\title{
NONLOCAL CAUCHY PROBLEM FOR TIME VARYING DELAY INTEGRODIFFERENTIAL EQUATIONS OF SOBOLEV TYPE IN BANACH SPACES
}

\author{
K. BALACHANDRAN AND R. RAVIKUMAR
}

\begin{abstract}
In this paper we prove the existence of mild and strong solutions of nonlinear time varying delay integrodifferential equations of Sobolev type with nonlocal conditions in Banach spaces. The results are obtained by using the theory of compact semigroups and Schaefer's fixed point theorem.
\end{abstract}

\section{Introduction}

Several papers have appeared on the problem of existence of solutions of semilinear differential equations and integrodifferential equations in Banach spaces [1-3, 11-12, 14-16]. Byszewski [6] has established the existence and uniqueness of mild, strong and classical solutions of the semilinear nonlocal Cauchy problem in a Banach space by using semigroup theroy and the contraction mapping principle. Subsequently he has investigated the same problem for different types of evolution equations in Banach spaces [7-10]. Many papers have been appeared on nonlocal Cauchy problem for various classes of differential and integrodifferential equations [13-16]. Physical motivation for this kind of problem is given in [6-10].

Brill [5] investigated the existence of solutions for a semilinear Sobolev evolution equation in a Banach space. This type of equations arise in various applications such as in the flow of fluid through fissured rocks, thermodynamics and shear in second order fluids (see [5]). Recently, Balachandran et al [3, 4] discussed the problem for nonlinear integrodifferential equations of Sobolev type with nonlocal conditions in Banach spaces. In this paper, we shall establish the existence of solutions of time varying delay integrodifferential equations of Sobolev type with nonlocal conditions by using the compact semigroup and the Schaefer fixed point theorem.

Received January 12, 2005.

2000 Mathematics Subject Classification. 34G20, 47H20.

Key words and phrases. Existence theorem, delay integrodifferential equations, fixed point theorem, nonlocal condition. 


\section{Basic Assumptions}

Consider the nonlinear delay integrodifferential equation of Sobolev type with nonlocal condition of the form

$$
\begin{gathered}
(E u(t))^{\prime}+A u(t)=F\left(t, u\left(\sigma_{1}(t)\right), \int_{0}^{t} g\left(t, s, u\left(\sigma_{2}(s)\right), \int_{0}^{s} k\left(s, \tau, u\left(\sigma_{3}(\tau)\right)\right) d \tau\right) d s\right) \\
u(0)+q(u(\cdot))=u_{0} \text { for } 0 \leq t \leq b,
\end{gathered}
$$

where $F: J \times X \times X \rightarrow Y, g: J \times J \times X \times X \rightarrow X, k: J \times J \times X \rightarrow X$ and $q: X \rightarrow X$ are given functions. Moreover, $\sigma_{i}: J \rightarrow J, i=1,2,3$ are continuous function such that $\sigma_{i}(t) \leq t, i=1,2,3$ and $u_{0} \in D(E)$. Let $J=[0, b]$ and we assume the following conditions:

(i) For each $t \in J$, the function $F(t, \cdot, \cdot): X \times X \rightarrow Y$ is continuous and for each $x, y \in X$, the function $F(\cdot, x, y): J \rightarrow Y$ is strongly measurable.

(ii) For each $t, s \in J$, the function $g(t, s, \cdot, \cdot): X \times X \rightarrow X$ is continuous and for each $x, y \in X$, the function $g(\cdot, \cdot, x, y): J \times J \rightarrow X$ is strongly measurable.

(iii) For each $t, s \in J$, the function $k(t, s, \cdot): X \rightarrow X$ is continuous and for each $z \in X$, the function $k(\cdot, \cdot, z): J \times J \rightarrow X$ is strongly measurable.

(iv) For each positive integer $r$, there exists $h_{r} \in L^{1}([0, b])$ such that

$$
\sup _{\|x\|,|y| \leq r}\left\|F\left(t, u\left(\sigma_{1}(t)\right), \int_{0}^{t} g\left(t, s, u\left(\sigma_{2}(s)\right), \int_{0}^{s} k\left(s, \tau, u\left(\sigma_{3}(\tau)\right)\right) d \tau\right) d s\right)\right\| \leq h_{r}(t)
$$

Definition 2.1. A continuous solution $u(t)$ of the integral equation

$$
\begin{aligned}
u(t)= & E^{-1} T(t) E u_{0}-E^{-1} T(t) E q(u(\cdot)) \\
& +\int_{0}^{t} E^{-1} T(t-s) F\left(s, u\left(\sigma_{1}(s)\right), \int_{0}^{s} g\left(s, \tau, u\left(\sigma_{2}(\tau)\right), \int_{0}^{\tau} k\left(\tau, \theta, u\left(\sigma_{3}(\theta)\right)\right) d \theta\right) d \tau\right) d s
\end{aligned}
$$

is called a mild solution of problem (1)-(2) on $J$.

Definition 2.2. A function $u$ is said to be a strong solution of problem (1), (2) on $J$ if $u$ is differentiable almost every where on $J, u^{\prime}(t) \in L^{1}(J, X), u(0)+q(u(\cdot))=u_{0}$ and

$$
(E u(t))^{\prime}+A u(t)=F\left(t, u\left(\sigma_{1}(t)\right), \int_{0}^{t} g\left(t, s, u\left(\sigma_{2}(s)\right), \int_{0}^{s} k\left(s, \tau, u\left(\sigma_{3}(\tau)\right)\right) d \tau\right) d s\right)
$$

almost everywhere on $J$.

In order to prove our main theorem, we assume certain conditions on the operators $A$ and $E$. Let $X$ and $Y$ be Banach spaces with norm $|\cdot|$ and $\|\cdot\|$, respectively. The operators $A: D(A) \subset X \rightarrow Y$ and $E: D(E) \subset X \rightarrow Y$ satisfy the following hypothesis.

$\left(\mathrm{H}_{1}\right) A$ and $E$ are closed linear operators.

$\left(\mathrm{H}_{2}\right) D(E) \subset D(A)$ and $E$ is bijective. 
$\left(\mathrm{H}_{3}\right) E^{-1}: Y \rightarrow D(E)$ is continuous.

Hypothesis $\left(\mathrm{H}_{1}\right)$ and $\left(\mathrm{H}_{2}\right)$ and the closed graph theorem imply the boundedness of the linear operator $A E^{-1}: Y \rightarrow Y$ and $-A E^{-1}$ generates a uniformly continuous semigroup $T(t), t \geq 0$, of bounded linear operators from $Y$ into $Y$.

$\left(\mathrm{H}_{4}\right)$ For some $\lambda \in \rho\left(-A E^{-1}\right)$, the resolvent set of $-A E^{-1}$, the resolvent $R\left(\lambda,-A E^{-1}\right)$ is a compact operator.

Let $T(t)$ be a uniformly continuous semigroup and let $A$ be its infinitesimal generator. If the resolvent set $R(\lambda: A)$ of $A$ is compact for every $\lambda \in \rho(A)$, then $T(t)$ is compact semigroup [17].

From the above fact that $-A E^{-1}$ generates a compact semigroup $T(t), t>0$, and so $\max _{t \in J}\|T(t)\|$ is finite and denote $\alpha=\left\|E^{-1}\right\|$. We need the following fixed point theorem to prove our results.

Schaefer's Theorem.([18]) Let $Z$ be a normed linear space. Let $\Phi: Z \rightarrow Z$ be a completely continuous operator, that is, it is continuous and the image of any bounded set is contained in a compact set, and let

$$
\zeta(\Phi)=\{x \in Z: x=\lambda \Phi x \text { for some } 0<\lambda<1\} .
$$

Then either $\zeta(\Phi)$ is unbounded or $\Phi$ has a fixed point.

\section{Existence of a Mild Solution}

Theorem 3.1. Let $F: J \times X \times X \rightarrow Y, g: J \times J \times X \times X \rightarrow X$ and $k: J \times J \times X \rightarrow X$ be functions satisfying Conditions (i)-(iv). Assume that $\left(H_{1}\right)-\left(H_{4}\right)$ hold. Further assume that:

(v) There exists a continuous function $m_{1}: J \rightarrow[0, \infty)$ and such that

$$
\|F(t, x, y)\| \leq m_{1}(t) \Omega_{2}(\|x\|+\|y\|), \quad 0 \leq t \leq b, \quad x, y \in X,
$$

where $\Omega_{2}:[0, \infty) \rightarrow(0, \infty)$ is a continuous nondecreasing function.

(vi) There exists a continuous function $m_{2}: J \times J \rightarrow[0, \infty)$ such that

$$
\|g(t, s, x, y)\| \leq m_{2}(t, s) \Omega_{1}(\|x\|+\|y\|), \quad 0 \leq s \leq t \leq b, \quad x, y \in X
$$

where $\Omega_{1}:[0, \infty) \rightarrow(0, \infty)$ is a continuous nondecreasing function.

(vii) There exists a continuous function $m_{3}: J \times J \rightarrow[0, \infty)$ such that

$$
\|k(t, s, z)\| \leq m_{3}(t, s) \Omega_{0}(\|z\|), \quad 0 \leq s \leq t \leq b, \quad z \in X,
$$

where $\Omega_{0}:[0, \infty) \rightarrow(0, \infty)$ is a continuous nondecreasing function.

(viii) $T(t)$ is a compact semigroup and there exists a constant $M>0$ such that

$$
\|T(t)\| \leq M
$$


(ix) $q: C(J: X) \rightarrow D(E) \subset X$, is continuous, compact and there exists a constant $H>0$ such that

$$
\|E q(u(\cdot))\| \leq H, \quad \text { for } \quad u \in C(J: X) .
$$

Further if

$$
\int_{0}^{b} m^{*}(s) d s<\int_{c}^{\infty} \frac{d s}{2 \Omega_{0}(s)+2 \Omega_{1}(s)+\Omega_{2}(s)}
$$

where $c=\alpha M\left(\left\|E u_{0}\right\|+H\right)$ and

$$
m^{*}(t)=\max \left\{\alpha M m_{1}(t), m_{2}(t, t)+\int_{0}^{t} \frac{\partial m_{2}(t, s)}{\partial t} d s, m_{3}(t, t)+\int_{0}^{t} \frac{\partial m_{3}(t, s)}{\partial t} d s\right\},
$$

then the problem (1)-(2) has atleast one mild solution on $J$.

Proof. Let $B=C(J, X)$. We establish the existence of a mild solution of the problem (1)-(2) by applying the Schaefer fixed point theorem. First we obtain a priori bounds for the operator equation

$$
u(t)=\lambda \Phi u(t), \quad 0<\lambda<1 .
$$

where $\Phi: B \rightarrow B$ is defined by

$$
\begin{aligned}
\Phi u(t) & =E^{-1} T(t) E u_{0}-E^{-1} T(t) E q(u(\cdot)) \\
& +\int_{0}^{t} E^{-1} T(t-s) F\left(s, u\left(\sigma_{1}(s)\right), \int_{0}^{s} g\left(s, \tau, u\left(\sigma_{2}(\tau)\right), \int_{0}^{\tau} k\left(\tau, \theta, u\left(\sigma_{3}(\theta)\right)\right) d \theta\right) d \tau\right) d s .
\end{aligned}
$$

Then from (3)-(4) we have

$$
\begin{aligned}
|u(t)| \leq & \alpha M\left(\left\|E u_{0}\right\|+H\right)+\alpha M \int_{0}^{t} \| F\left(s, u\left(\sigma_{1}(s)\right), \int_{0}^{s} g\left(s, \tau, u\left(\sigma_{2}(\tau)\right),\right.\right. \\
& \left.\left.\int_{0}^{\tau} k\left(\tau, \theta, u\left(\sigma_{3}(\theta)\right)\right) d \theta\right) d \tau\right) \| d s \\
\leq & \alpha M\left(\left\|E u_{0}\right\|+H\right)+\alpha M \int_{0}^{t}\left\{m _ { 1 } ( s ) \Omega _ { 2 } \left[\left\|u\left(\sigma_{1}(s)\right)\right\|+\int_{0}^{s} m_{1}(s, \tau) \Omega_{1}\left(\left\|u\left(\sigma_{2}(\tau)\right)\right\|\right.\right.\right. \\
& \left.\left.\left.+\int_{0}^{\tau} \Omega_{0}\left(\left\|u\left(\sigma_{3}(\theta)\right)\right\|\right) d \theta\right) d \tau\right]\right\} d s .
\end{aligned}
$$

Let us take the right hand side of the above inequality as $v(t)$. Then we have

$$
\begin{gathered}
v(0)=u(0)=\alpha M\left(\left\|E u_{0}\right\|+H\right), \text { and }|u(t)| \leq v(t) \\
v^{\prime}(t)=\alpha M\left\{m _ { 1 } ( t ) \Omega _ { 2 } \left[\left\|u\left(\sigma_{1}(t)\right)\right\|+\int_{0}^{t} m_{2}(t, s) \Omega_{1}\left(\left\|u\left(\sigma_{2}(s)\right)\right\|\right.\right.\right. \\
\left.\left.\left.+\int_{0}^{s} m_{3}(s, \tau) \Omega_{0}\left(\left\|u\left(\sigma_{3}(\tau)\right)\right\|\right) d \tau\right) d s\right]\right\} \\
\leq \alpha M m_{1}(t) \Omega_{2}\left[v(t)+\int_{0}^{t} m_{2}(t, s) \Omega_{1}\left(v(s)+\int_{0}^{s} m_{3}(s, \tau) \Omega_{0}(v(\tau)) d \tau\right) d s\right]
\end{gathered}
$$


since $v$ is obviously increasing and $\sigma_{i}(t) \leq t$ for $\mathrm{i}=1,2,3$.

$$
\text { Let } w(t)=v(t)+\int_{0}^{t} m_{2}(t, s) \Omega_{1}\left(v(s)+\int_{0}^{s} m_{3}(s, \tau) \Omega_{0}(v(\tau)) d \tau\right) d s
$$

Then

$$
\begin{gathered}
w(0)=v(0)=c, \quad v(t) \leq w(t) \\
w^{\prime}(t)=v^{\prime}(t)+m_{2}(t, t) \Omega_{1}\left(v(t)+\int_{0}^{t} m_{3}(t, s) \Omega_{0}(v(s)) d s\right) \\
+\int_{0}^{t} \frac{\partial m_{2}(t, s)}{\partial t} \Omega_{1}\left(v(s)+\int_{0}^{s} m_{3}(s, \tau) \Omega_{0}(v(\tau)) d \tau\right) d s \\
\leq \alpha M m_{1}(t) \Omega_{2}(w(t))+m_{2}(t, t) \Omega_{1}\left(w(t)+\int_{0}^{t} m_{3}(t, s) \Omega_{0}(w(s)) d s\right) \\
+\int_{0}^{t} \frac{\partial m_{2}(t, s)}{\partial t} \Omega_{1}\left(w(s)+\int_{0}^{s} m_{3}(s, \tau) \Omega_{0}(w(\tau)) d \tau\right) d s .
\end{gathered}
$$

Let $r(t)=w(t)+\int_{0}^{t} m_{3}(t, s) \Omega_{0}(w(s)) d s$,

then $r(0)=w(0)=c, \quad w(t) \leq r(t)$

$$
\begin{aligned}
r^{\prime}(t)= & w^{\prime}(t)+m_{3}(t, t) \Omega_{0}(w(t))+\int_{0}^{t} \frac{\partial m_{3}(t, s)}{\partial t} \Omega_{0}(w(s)) d s \\
\leq & \alpha M m_{1}(t) \Omega_{2}(r(t))+m_{2}(t, t) \Omega_{1}(r(t))+\int_{0}^{t} \frac{\partial m_{2}(t, s)}{\partial t} \Omega_{1}(r(s)) d s \\
& +m_{3}(t, t) \Omega_{0}(r(t))+\int_{0}^{t} \frac{\partial m_{3}(t, s)}{\partial t} \Omega_{0}(r(s)) d s \\
\leq & \alpha M m_{1}(t) \Omega_{2}(r(t))+m_{2}(t, t) \Omega_{1}(r(t))+\int_{0}^{t} \frac{\partial m_{2}(t, s)}{\partial t} d s \Omega_{1}(r(t)) \\
& +m_{3}(t, t) \Omega_{0}(r(t))+\int_{0}^{t} \frac{\partial m_{3}(t, s)}{\partial t} d s \Omega_{0}(r(t))
\end{aligned}
$$

This implies

$$
\int_{r(0)}^{r(t)} \frac{d s}{2 \Omega_{0}(s)+2 \Omega_{1}(s)+\Omega_{2}(s)} \leq \int_{0}^{b} m^{*}(s) d s<\int_{c}^{\infty} \frac{d s}{2 \Omega_{0}(s)+2 \Omega_{1}(s)+\Omega_{2}(s)} .
$$

This inequality implies that there exists a constant $K$ such that $r(t) \leq K, t \in J$, and hence $u(t) \leq K$ where $K$ depends only on $b$ and on the functions $m_{1}, m_{2}, m_{3}, \Omega_{0}, \Omega_{1}$ and $\Omega_{2}$.

Next we prove that the operator $\Phi: B \rightarrow B$ defined by

$$
\begin{aligned}
(\Phi y)(t) & =E^{-1} T(t) E u_{0}-E^{-1} T(t) E q(u(\cdot)) \\
& +\int_{0}^{t} E^{-1} T(t-s) F\left(s, u\left(\sigma_{1}(s)\right), \int_{0}^{s} g\left(s, \tau, u\left(\sigma_{2}(\tau)\right), \int_{0}^{\tau} k\left(\tau, \theta, u\left(\sigma_{3}(\theta)\right)\right) d \theta\right) d \tau\right) d s
\end{aligned}
$$


is a completely continuous operator.

Let $B_{r}=\{y \in B:\|y\| \leq r\}$ for some $r \geq 1$. We first show that $\Phi$ maps $B_{r}$ into an equicontinuous family. Let $y \in B_{r}$ and $t_{1}, t_{2} \in J$ and $\epsilon>0$. Then if $0<\epsilon<t_{1}<t_{2} \leq b$,

$$
\begin{aligned}
& \left\|(\Phi y)\left(t_{1}\right)-(\Phi y)\left(t_{2}\right)\right\| \\
\leq & \alpha\left\|T\left(t_{1}\right)-T\left(t_{2}\right)\right\|\left(\left\|E u_{0}\right\|+H\right)+\alpha \int_{0}^{t_{1}}\left\|\left(T\left(t_{1}-s\right)-T\left(t_{2}-s\right)\right)\right\| \\
& \times\left\|F\left(s, u\left(\sigma_{1}(s)\right), \int_{0}^{s} g\left(s, \tau, u\left(\sigma_{2}(\tau)\right), \int_{0}^{\tau} k\left(\tau, \theta, u\left(\sigma_{3}(\theta)\right)\right) d \theta\right) d \tau\right)\right\| d s \\
& +\alpha \int_{t_{1}}^{t_{2}}\left\|T\left(t_{2}-s\right)\right\|\left\|F\left(s, u\left(\sigma_{1}(s)\right), \int_{0}^{s} g\left(s, \tau, u\left(\sigma_{2}(\tau)\right), \int_{0}^{\tau} k\left(\tau, \theta, u\left(\sigma_{3}(\theta)\right)\right) d \theta\right) d \tau\right)\right\| d s \\
\leq & \alpha\left\|T\left(t_{1}\right)-T\left(t_{2}\right)\right\|\left(\left\|E u_{0}\right\|+H\right)+\alpha \int_{0}^{t_{1}}\left\|\left(T\left(t_{1}-s\right)-T\left(t_{2}-s\right)\right)\right\| h_{r}(s) d s \\
& +\alpha \int_{t_{1}}^{t_{2}}\left\|T\left(t_{2}-s\right)\right\| h_{r}(s) d s .
\end{aligned}
$$

As $t_{2}-t_{1} \rightarrow 0$, the right-hand side of the above inequality tends to zero since the compactness of $T(t)$ for $t>0$ implies the continuity in the uniform operator topology. Thus $\Phi$ maps $B_{r}$ into an equicontinuous family of functions. It is easy to see that the family $\Phi B_{r}$ is uniformly bounded.

Next we show that $\overline{\Phi B_{r}}$ is compact. Since we have proved that $\Phi B_{r}$ is an equicontinuous family, it is sufficient, by the Arzela-Ascoli theorem, to show that $\Phi$ maps $B_{r}$ into a precompact set in $X$. This is clear when $t=0$, the set $\Phi y(0)=\left\{u_{0}-q(y)\right\}$ is precompact in $X$, since $q$ is compact.

Let $0<t \leq b$ be fixed and $\epsilon$ a real number satisfying $0<\epsilon<t$. For $y \in B_{r}$, we define

$$
\begin{aligned}
\left(\Phi_{\epsilon} y\right)(t) & =E^{-1} T(t) E u_{0}-E^{-1} T(t) E q(u(\cdot)) \\
& +\int_{0}^{t} E^{-1} T(t-s) F\left(s, u\left(\sigma_{1}(s)\right), \int_{0}^{s} g\left(s, \tau, u\left(\sigma_{2}(\tau)\right), \int_{0}^{\tau} k\left(\tau, \theta, u\left(\sigma_{3}(\theta)\right)\right) d \theta\right) d \tau\right) d s .
\end{aligned}
$$

Since $T(t)$ is a compact operator, the set $Y_{\epsilon}(t)=\left\{\left(\Phi_{\epsilon} y\right)(t): y \in B_{r}\right\}$ is precompact in $X$, for every $\epsilon, 0<\epsilon<t$. Moreover for every $y \in B_{r}$, we have

$$
\begin{aligned}
& \left\|(\Phi y)(t)-\left(\Phi_{\epsilon} y\right)(t)\right\| \\
& \leq \alpha \int_{t-\epsilon}^{t}\left\|T(t-s) F\left(s, u\left(\sigma_{1}(s)\right), \int_{0}^{s} g\left(s, \tau, u\left(\sigma_{2}(\tau)\right), \int_{0}^{\tau} k\left(\tau, \theta, u\left(\sigma_{3}(\theta)\right)\right) d \theta\right) d \tau\right)\right\| d s \\
& \leq \alpha M \int_{t-\epsilon}^{t} h_{r}(s) d s .
\end{aligned}
$$

Therefore there are precompact sets arbitrarily close to the set $\left\{(\Phi y)(t): y \in B_{r}\right\}$. Hence the set $\left\{(\Phi y)(t): y \in B_{r}\right\}$ is precompact in $X$. 
It remains to show that $\Phi: B \rightarrow B$ is continuous. Let $\left\{u_{j}\right\}$ be a sequence such that $u_{j} \rightarrow u$ in $B$. Then there is an integer $l$ such that $\left\|u_{j}\right\| \leq l$ for all $j$ and $\|u\| \leq l, \mathrm{t} \in J$ and so $u_{j} \in B_{l}$ and $u \in B_{l}$. By (i) and (ii)

$$
\begin{aligned}
F\left(s, u_{j}\left(\sigma_{1}(s)\right),\right. & \left.\int_{0}^{s} g\left(s, \tau, u_{j}\left(\sigma_{2}(\tau)\right), \int_{0}^{\tau} k\left(\tau, \theta, u_{j}\left(\sigma_{3}(\theta)\right)\right) d \theta\right) d \tau\right) \\
& \rightarrow F\left(s, u\left(\sigma_{1}(s)\right), \int_{0}^{s} g\left(s, \tau, u\left(\sigma_{2}(\tau)\right), \int_{0}^{\tau} k\left(\tau, \theta, u\left(\sigma_{3}(\theta)\right)\right) d \theta\right) d \tau\right)
\end{aligned}
$$

for each $t \in J$ and since

$$
\begin{aligned}
& \| F\left(s, u_{j}\left(\sigma_{1}(s)\right), \int_{0}^{s} g\left(s, \tau, u_{j}\left(\sigma_{2}(\tau)\right), \int_{0}^{\tau} k\left(\tau, \theta, u_{j}\left(\sigma_{3}(\theta)\right)\right) d \theta\right) d \tau\right) \\
& \rightarrow F\left(s, u\left(\sigma_{1}(s)\right), \int_{0}^{s} g\left(s, \tau, u\left(\sigma_{2}(\tau)\right), \int_{0}^{\tau} k\left(\tau, \theta, u\left(\sigma_{3}(\theta)\right)\right) d \theta\right) d \tau\right) \| \leq 2 h_{l}(t),
\end{aligned}
$$

we have by dominated convergence theorem,

$$
\begin{aligned}
\| & u_{j}-\Phi u \| \\
= & \sup _{t \in J} \| \int_{0}^{t} E^{-1} T(t-s)\left\{F\left(s, u_{j}\left(\sigma_{1}(s)\right), \int_{0}^{s} g\left(s, \tau, u_{j}\left(\sigma_{2}(\tau)\right), \int_{0}^{\tau} k\left(\tau, \theta, u_{j}\left(\sigma_{3}(\theta)\right)\right) d \theta\right) d \tau\right)\right. \\
& \left.-F\left(s, u\left(\sigma_{1}(s)\right), \int_{0}^{s} g\left(s, \tau, u\left(\sigma_{2}(\tau)\right), \int_{0}^{\tau} k\left(\tau, \theta, u\left(\sigma_{3}(\theta)\right)\right) d \theta\right) d \tau\right)\right\} d s \| \\
\leq & \alpha M \int_{0}^{t} \| F\left(s, u_{j}\left(\sigma_{1}(s)\right), \int_{0}^{s} g\left(s, \tau, u_{j}\left(\sigma_{2}(\tau)\right), \int_{0}^{\tau} k\left(\tau, \theta, u_{j}\left(\sigma_{3}(\theta)\right)\right) d \theta\right) d \tau\right) \\
& -F\left(s, u\left(\sigma_{1}(s)\right), \int_{0}^{s} g\left(s, \tau, u\left(\sigma_{2}(\tau)\right), \int_{0}^{\tau} k\left(\tau, \theta, u\left(\sigma_{3}(\theta)\right)\right) d \theta\right) d \tau\right) \| d s \rightarrow 0, \text { as } j \rightarrow \infty .
\end{aligned}
$$

Thus $\Phi$ is continuous. This complete the proof that $\Phi$ is completely continuous.

We have already proved that the set $\zeta(\Phi)=\{y \in B: y=\lambda \Phi y, \lambda \in(0,1)\}$ is bounded and, by Schaefer's theorem, the operator $\Phi$ has a fixed point in $B$. This means that the problem (1)-(2) has a mild solution.

\section{Example}

Consider the partial integrodifferential equation of the form

$$
\begin{aligned}
& \frac{\partial}{\partial t}\left[z(t, y)-z_{y y}(t, y)\right] \\
& =\frac{\partial^{2}}{\partial y^{2}} z(t, y)+\frac{z^{2}(t, y) \sin (z(t, y))}{(1+t)\left(1+t^{2}\right)}+\int_{0}^{t}\left[\frac{z(s, y)}{(1+t)\left(1+t^{2}\right)^{2}(1+s)^{2}}\right. \\
& \left.\quad+\frac{1}{(1+t)\left(1+t^{2}\right)} \int_{0}^{s} \frac{z(\tau, y)}{(1+s)(1+\tau)} \exp z(\tau, y) d \tau\right] d s
\end{aligned}
$$




$$
\begin{gathered}
z(t, 0)=z(t, 1)=0, t \in J=[0,1] \\
z(0, y)-\sum_{i=1}^{p} z\left(t_{i}, y\right)=z_{0}(y), 0<y<1 ; 0<t_{1}<t_{2}<\cdots<t_{p}<1 .
\end{gathered}
$$

Let $X$ denote the Banach space $L^{2}([0, \pi])$ and $z(t, y)=u(t)(y)$.

Let

$$
q(u(\cdot))=\sum_{i=1}^{p} u\left(t_{i}\right) .
$$

We can easily check that there exists $H>0$ such that

$$
\|q(u(\cdot))\| \leq H
$$

for instance we may take $H=p r$, if $\|u(t)\| \leq r$. On the other hand,we have

$$
\left\|q\left(u_{1}(\cdot)\right)-q\left(u_{2}(\cdot)\right)\right\| \leq p\left\|u_{1}(t)-u_{2}(t)\right\| .
$$

Moreover, since

$$
\begin{gathered}
F\left(t, u\left(\sigma_{1}(t)\right), \int_{0}^{t} g\left(t, s, u\left(\sigma_{2}(s)\right), \int_{0}^{s} k\left(s, \tau, u\left(\sigma_{3}(\tau)\right)\right) d \tau\right) d s\right) \\
=\frac{u^{2}(t-\sin t) \sin (u(t-\sin t))}{(1+t)\left(1+t^{2}\right)}+\int_{0}^{t}\left[\frac{u(s-\sin s)}{(1+t)\left(1+t^{2}\right)^{2}(1+s)^{2}}\right. \\
\left.\quad+\frac{1}{(1+t)\left(1+t^{2}\right)} \int_{0}^{s} \frac{u(\tau-\sin \tau)}{(1+s)(1+\tau)} \exp u(\tau-\sin \tau) d \tau\right] d s,
\end{gathered}
$$

then,

$$
\begin{aligned}
\|F(t, u, z)\| & =\left\|\frac{1}{(1+t)\left(1+t^{2}\right)}\left(u^{2}(t-\sin t) \sin (u(t-\sin t))+z\right)\right\| \\
& \leq \frac{1}{\left(1+t^{2}\right)}\|u\|^{2}+\frac{1}{(1+t)}\|z\|
\end{aligned}
$$

where we have set

$$
z=\int_{0}^{t} g\left(t, s, u\left(\sigma_{2}(s)\right), \int_{0}^{s} k\left(s, \tau, u\left(\sigma_{3}(\tau)\right)\right) d \tau\right) d s
$$

Next if

$$
v=\int_{0}^{s} k\left(s, \tau, u\left(\sigma_{3}(\tau)\right) d \tau\right.
$$

then,

$$
\begin{aligned}
\|g(t, s, u, v)\| & =\left\|\frac{u(s-\sin s)}{(1+t)\left(1+t^{2}\right)^{2}(1+s)^{2}}+\frac{v}{(1+t)\left(1+t^{2}\right)}\right\| \\
& \leq \frac{\|u(s-\sin s)\|}{\left(1+t^{2}\right)^{2}(1+s)}+\frac{1}{\left(1+t^{2}\right)(1+t)}\|v\| .
\end{aligned}
$$


Finally, we have

$$
\begin{aligned}
k(s, \tau, u) & =\left\|\frac{u(\tau-\sin \tau) \exp (u(\tau-\sin \tau))}{(1+s)(1+\tau)}\right\| \\
& \leq \frac{1}{(1+s)(1+\tau)}\|u(\tau-\sin \tau)\| \exp (\|u(\tau-\sin \tau)\|) .
\end{aligned}
$$

Define the operators $A: D(A) \subset X \rightarrow Y$ and $E: D(E) \subset X \rightarrow Y$ by

$$
A w=w^{\prime \prime}, \text { and } E w=w-w^{\prime \prime},
$$

where each domain $D(A)$ and $D(E)$ is given by

$$
\left\{w \in X: \quad w, w^{\prime} \text { are absolutely continuous, } w^{\prime \prime} \in X, w(0)=w(\pi)=0\right\} .
$$

Then $A$ and $E$ can be written, respectively, as

$$
\begin{aligned}
& A w=\sum_{n=1}^{\infty} n^{2}\left(w, w_{n}\right) w_{n}, \quad w \in D(A), \\
& E w=\sum_{n=1}^{\infty}\left(1+n^{2}\right)\left(w, w_{n}\right) w_{n}, \quad w \in D(E),
\end{aligned}
$$

where $w_{n}(x)=\left\{\frac{\sqrt{2}}{\pi} \sin n s\right\}_{n \geq 1}$, is the orthogonal set of eigenvectors of $A$. Furthermore for $w \in X$ we have

$$
\begin{aligned}
E^{-1} w & =\sum_{n=1}^{\infty} \frac{1}{1+n^{2}}\left(w, w_{n}\right) w_{n}, \\
-A E^{-1} w & =\sum_{n=1}^{\infty} \frac{-n^{2}}{1+n^{2}}\left(w, w_{n}\right) w_{n}, \\
T(t) w & =\sum_{n=1}^{\infty} \exp \left(\frac{-n^{2} t}{1+n^{2}}\right)\left(w, w_{n}\right) w_{n} .
\end{aligned}
$$

It is easy to see that $A E^{-1}$ generates a strongly continuous semigroup $T(t)$ on $Y$ and $T(t)$ is compact such that $|T(t)| \leq e^{-t}$ for each $t>0$. Further we have,

$$
\begin{aligned}
& \mid \frac{1}{(1+t)\left(1+t^{2}\right)}\left[z^{2}(\sin t, y) \sin z(t, y)+\int_{0}^{t}\left(\frac{z(s, y)}{\left(1+t^{2}\right)(1+s)^{2}}\right.\right. \\
& \left.\left.\quad+\int_{0}^{s} \frac{z(\tau, y)}{(1+s)(1+\tau)} \exp z(\sin \tau, y) d \tau\right) d s\right]\left|\leq \frac{1}{\left(1+t^{2}\right)}\right| z \mid
\end{aligned}
$$

Moreover all the other conditions stated in Theorem 3.1 are satisfied. Hence the equation (5) has a mild solution on $[0,1]$. 


\section{Existence of a Strong Solution}

Theorem 5.1. Let assumption (i)-(ix) in Theorem 3.1 be satisfied and the following additional assumptions hold:

(x) $Y$ is a reflexive Banach space and $B_{r}=\{y \in B:\|y\| \leq r\}$.

(xi) $F: J \times X \times X \rightarrow Y$ is continuous in $t$ on $J$ and there exists constants $N_{0}>0$ and $N>0$ such that

$$
\begin{gathered}
\|F(t, u, v)\| \leq N_{0}, \\
\left\|F(t, u, v)-F\left(s, u_{1}, v_{1}\right)\right\| \leq N\left[|t-s|+\left\|u-u_{1}\right\|+\left\|v-v_{1}\right\|\right], \\
\text { for } t, s \in J \text { and } u, u_{1}, v, v_{1} \in B_{r} .
\end{gathered}
$$

(xii) $g: J \times J \times X \times X \rightarrow X$ is continuous in $t$ on $J$ and there exists constants $N_{1}>0$ and $N_{2}>0$ such that

$$
\begin{aligned}
& \|g(s, \tau, u, v)\| \leq N_{1}, \\
& \|g(t, \tau, u, v)-g(s, \tau, u, v)\| \leq N_{2}[|t-s|], \\
& \quad \text { for } t, s, \tau \in J \text { and } u, v \in B_{r} .
\end{aligned}
$$

(xiii) $u$ is the unique mild solution of problem (1)-(2) and there is a constant $\gamma$ such that

$$
\left\|u\left(\sigma_{1}(t)\right)-u\left(\sigma_{1}(s)\right)\right\| \leq \gamma\|u(t)-u(s)\|, \text { for } t, s \in J .
$$

Then $u$ is the unique strong solution of the problem (1)-(2) on J.

Proof. Since all the assumptions of the Theorem 3.1 are satisfied, then the problem (1)-(2) possesses a mild solution $u$ which, according to assumption (xiii), is the unique mild solution of the problem (1)-(2).

Now we show that this mild solution is the unique strong solution of the problem (1)-(2) on $J$. For any $t \in J$, we have

$$
\begin{aligned}
& u(t+h)-u(t) \\
& =E^{-1}[T(t+h)-T(t)] E u_{0}-E^{-1}[T(t+h)-T(t)] E q(u(\cdot)) \\
& \quad+\int_{0}^{h} E^{-1} T(t+h-s) F\left(s, u\left(\sigma_{1}(s)\right), \int_{0}^{s} g\left(s, \tau, u\left(\sigma_{2}(\tau)\right), \int_{0}^{\tau} k\left(\tau, \theta, u\left(\sigma_{3}(\theta)\right)\right) d \theta\right) d \tau\right) d s \\
& \quad+\int_{h}^{t+h} E^{-1} T(t+h-s) F\left(s, u\left(\sigma_{1}(s)\right), \int_{0}^{s} g\left(s, \tau, u\left(\sigma_{2}(\tau)\right), \int_{0}^{\tau} k\left(\tau, \theta, u\left(\sigma_{3}(\theta)\right)\right) d \theta\right) d \tau\right) d s \\
& \quad-\int_{0}^{t} E^{-1} T(t-s) F\left(s, u\left(\sigma_{1}(s)\right), \int_{0}^{s} g\left(s, \tau, u\left(\sigma_{2}(\tau)\right), \int_{0}^{\tau} k\left(\tau, \theta, u\left(\sigma_{3}(\theta)\right)\right) d \theta\right) d \tau\right) d s .
\end{aligned}
$$


From our assumptions, we have

$$
\begin{aligned}
& \|u(t+h)-u(t)\| \\
& \leq \alpha\|T(t+h)-T(t)\|\left(\left\|E u_{0}\right\|+H\right)+\alpha M N_{0} h \\
& \quad+\int_{0}^{t} \| E^{-1} T(t-s)\left[F \left(s+h, u\left(\sigma_{1}(s+h)\right), \int_{0}^{s+h} g\left(s+h, \tau, u\left(\sigma_{2}(\tau)\right),\right.\right.\right. \\
& \left.\left.\left.\quad \int_{0}^{\tau} k\left(\tau, \theta, u\left(\sigma_{3}(\theta)\right)\right) d \theta\right) d \tau\right) d s\right), \\
& \left.\quad-F\left(s, u\left(\sigma_{1}(s)\right), \int_{0}^{s} g\left(s, \tau, u\left(\sigma_{2}(\tau)\right), \int_{0}^{\tau} k\left(\tau, \theta, u\left(\sigma_{3}(\theta)\right)\right) d \theta\right) d \tau\right)\right] d s \| \\
& \leq \alpha h M\left\|A E^{-1}\right\|\left(\left\|E u_{0}\right\|+H\right)+\alpha M N_{0} h+\alpha M N \int_{0}^{t}\left[h+\left\|u\left(\sigma_{1}(s+h)\right)-u\left(\sigma_{1}(s)\right)\right\|\right. \\
& \quad+\| \int_{0}^{s+h} g\left(s+h, \tau, u\left(\sigma_{2}(\tau)\right), \int_{0}^{\tau} k\left(\tau, \theta, u\left(\sigma_{3}(\theta)\right)\right) d \theta\right) d \tau \\
& \left.\quad-\int_{0}^{s} g\left(s, \tau, u\left(\sigma_{2}(\tau)\right), \int_{0}^{\tau} k\left(\tau, \theta, u\left(\sigma_{3}(\theta)\right)\right) d \theta\right) d \tau \|\right] d s \\
& \leq \alpha h M\left\|A E^{-1}\right\|\left(\left\|E u_{0}\right\|+H\right)+\alpha M N_{0} h \\
& \quad+\alpha M N \int_{0}^{t}\left[h+\gamma\|u(s+h)-u(s)\|+N_{1} h+N_{2} h b\right] d s \\
& \leq \alpha h M\left\|A E^{-1}\right\|\left(\left\|E u_{0}\right\|+H\right)+\alpha M N_{0} h+\alpha M N b h \\
& \quad+\alpha M N \gamma \int_{0}^{t}\|u(s+h)-u(s)\| d s+\alpha M N N_{1} b h+\alpha M N N_{2} b^{2} h \\
& \leq P h+\alpha M N \gamma \int_{0}^{t}\|u(s+h)-u(s)\| d s,
\end{aligned}
$$

where

$$
P=\alpha M\left\|A E^{-1}\right\|\left(\left\|E u_{0}\right\|+H\right)+\alpha M N_{0}+\alpha M N b+\alpha M N N_{1} b+\alpha M N N_{2} b^{2} .
$$

Using Gronwall's inequality, we get

$$
\|u(t+h)-u(t)\| \leq P h e^{\alpha M N b \gamma}, \quad t \in J
$$

Therefore $u$ is Lipschitz continuous on $J$.

The Lipschitz continuity of $u$ on $J$, combined with (u), gives that

$$
t \rightarrow F\left(t, u\left(\sigma_{1}(t)\right), \int_{0}^{t} g\left(t, s, u\left(\sigma_{2}(s)\right), \int_{0}^{s} k\left(s, \tau, u\left(\sigma_{3}(\tau)\right)\right) d \tau\right) d s\right)
$$

is Lipschitz continuous on $J$. Using the Corollary 2.11 in Section 4.2 in [7] and the definition of strong solution we observe that the linear Cauchy problem:

$$
(E v(t))^{\prime}+A v(t)=F\left(t, u\left(\sigma_{1}(t)\right), \int_{0}^{t} g\left(t, s, u\left(\sigma_{2}(s)\right), \int_{0}^{s} k\left(s, \tau, u\left(\sigma_{3}(\tau)\right)\right) d \tau\right) d s\right)
$$


$v(0)=u_{0}-q(u(\cdot))$, has a unique strong solution $v$ satisfying the equation

$$
\begin{aligned}
v(t)= & E^{-1} T(t) E u_{0}-E^{-1} T(t) E q(u(\cdot)) \\
& +\int_{0}^{t} E^{-1} T(t-s) F\left(s, u\left(\sigma_{1}(s)\right), \int_{0}^{s} g\left(s, \tau, u\left(\sigma_{2}(\tau)\right), \int_{0}^{\tau} k\left(\tau, \theta, u\left(\sigma_{3}(\theta)\right)\right) d \theta\right) d \tau\right) d s . \\
= & u(t) .
\end{aligned}
$$

Consequently $u$ is the unique strong solution of problem (1)-(2) on $J$.

\section{References}

[1] K. Balachandran, Existence and uniqueness of mild and strong solutions of nonlinear integrodifferential equation with nonlocal condition, Differential Equations and Dynamical Systems 6(1998), 159-165.

[2] K. Balachandran and S. Ilamaran, Existence and uniqueness of mild and strong solutions of a Volterra integrodifferential equation with nonlocal conditions, Tamkang Journal of Mathematics 28(1997), 93-100.

[3] K. Balachandran, J. Y. Park and M. Chandrasekaran, Nonlocal Cauchy Problem for delay integrodifferential equations of Sobolove type in Banach spaces, Applied Mathematics Letters 15(2002), 845-854.

[4] K. Balachandran, D. G. Park and Y. C. Kwun, Nonlinear integrodifferential equations of Sobolev type with nonlocal conditions in Banach spaces, Communications of the Korean Mathematical Society 14(1999), 223-231.

[5] H. Brill, A semilinear Sobolev evolution equation in a Banach space, Journal of Differential Equations 24(1977), 412-425.

[6] L. Byszewski, Theorems about the existence and uniqueness of solutions of a semilinear evolution nonlocal Cauchy problem, Journal of Mathematical Analysis and Applications 162(1991), 494-505.

[7] L. Byszewski, Existence, uniqueness and asymptotic stability of weak and strong solutions of functional- differential abstract nonlocal Cauchy problems, Dynamic Systems and Applications 5(1996), 595-606.

[8] L. Byszewski, On weak solutions of functional-differential abstract nonlocal Cauchy problems, Annales Polonici Mathematici 2(1997), 163-170.

[9] L. Byszewski, On a mild solution of a semilinear functional-differential evolution nonlocal problems, Journal of Applied Mathematics and Stochastic Analysis 10 (1997), 265-271.

[10] L. Byszewski and H. Akca, Existence of solutions of a semilinear functional differential evolution nonlocal problem, Nonlinear Analysis, Theory, Methods and Applications 34 (1998), 65-712.

[11] J. P. Dauer and K. Balachandran, Existence of solutions for an integrodifferential equation with nonlocal condition in Banach spaces, Libertas Mathematica 16 (1996), 133-143.

[12] S. Ilamaran and K. Balachandran, Existence and uniqueness of mild and strong solutions of an integrodifferential equation with nonlocal condition, Libertas Mathematica, 15(1995), 119-127.

[13] Y. Lin and J. H. Liu, Semilinear integrodifferential equations with nonlocal Cauchy problem, Nonlinear Analysis, Theory, Method and Applications, 26(1996), 1023-1033. 
[14] S. Mazouzi and N.-E. Tatar, Global existence for some integrodifferential equations with delay subject to nonlocal conditions, Journal for Analysis and its Applications, 21 (2002), 249-256.

[15] S. K. Ntouyas and P. Ch. Tsamatos, Global existence for semilinear evolution equations with nonlocal conditions, Journal of Mathematical Analysis and Applications, 210(1997), 679-987.

[16] S. K. Ntouyas and P. Ch. Tsamatos, Global existence for semilinear evolution integrodifferential equations with delay and nonlocal conditions, Applicable Analysis, 64 (1997), 99-105.

[17] A. Pazy, Semigroups of Linear Operators and Applications to Partial Differential Equations, Springer-Verlag, New York, 1983.

[18] H. Schaefer, Uber die methode der a priori schranken, Mathematische Annalem 129 (1955), 415-416.

Department of Mathematics, Bharathiar University, Coimbatore-641 046, India. 